\section{Transperineal ultrasonography for evaluation of the perianal fistula and abscess in pediatric Crohn disease: preliminary study}

Jae-Yeon Hwang ${ }^{1}$, Hye-Kyung Yoon ${ }^{2}$, Wee Kyoung Kim², Young Ah Cho², Jin Seong Lee², Chong Hyun Yoon ${ }^{2}$, Yeoun Joo Lee ${ }^{3}$, Kyung Mo Kim ${ }^{4}$

${ }^{1}$ Department of Radiology, Pusan National University Yangsan Hospital, Yangsan;

${ }^{2}$ Department of Radiology and Research Institute of Radiology, Asan Medical Center, University of Ulsan College of Medicine, Seoul; ${ }^{3}$ Department of Pediatrics, Pusan National University Children's Hospital, Yangsan; ${ }^{4}$ Department of Pediatrics, Asan Medical Center Children's Hospital, University of Ulsan College of Medicine, Seoul, Korea

Purpose: To assess the feasibility and effectiveness of transperineal ultrasonography (TPUS) for the evaluation of perianal Crohn disease (PCD) in pediatric patients.

Methods: Between September 2010 and August 2013, 64 TPUS examinations were performed in 43 patients (34 males and 9 females; mean age \pm standard deviation, 13.3 \pm 2.4 years; age range, 6 to 17 years) to evaluate PCD. The pain severity, location, and activity of perianal fistula, the presence of an abscess, and anal canal hyperemia were retrospectively evaluated. Spearman rank correlation analysis was performed to assess the relationship between the severity of the pain and the fistula activity, the presence of an abscess, and anal canal hyperemia.

Results: All examinations were successfully performed. Thirty-nine examinations (60.9\%) were performed without any pain experienced by the patient, 19 examinations (29.7\%) with mild pain, five examinations $(7.8 \%)$ with moderate pain, and one examination $(1.6 \%)$ with severe pain. The pain severity was correlated with the fistula activity $(P<0.01)$. An anterior fistula location was more common than a posterior location. Active fistulas and abscesses were identified during 30 examinations (46.9\%) and 12 examinations (18.8\%), respectively. Anal canal hyperemia was identified in 31 examinations (48.4\%).

Conclusion: TPUS with a color Doppler study is useful for visualizing a perianal fistula or abscess and for assessing its inflammatory activity in pediatric Crohn patients.

Keywords: Ultrasonography; Perineum; Crohn disease; Pediatrics

\section{Introduction}

Crohn disease (CD) is a chronic, inflammatory bowel disease of unknown etiology. Perianal Crohn disease $(P C D)$ refers to a refractory, inflammatory process involving perianal soft tissue, including

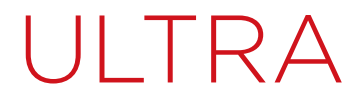

SONO

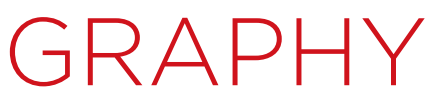

\section{ORIGINAL ARTICLE}

http://dx.doi.org/10.14366/usg. 14009 pISSN: 2288-5919 • elSSN: 2288-5943

Ultrasonography 2014;33:184-190

Received: January 27, 2014

Revised: March 5, 2014

Accepted: March 13, 2014

Correspondence to:

Hye-Kyung Yoon, MD, Department of Radiology and Research Institute of Radiology, Asan Medical Center, University of Ulsan College of Medicine, 88 Olympic-ro 43-gil, Songpa-gu, Seoul 138-736, Korea

Tel. +82-2-3010-5722

Fax. +82-2-476-0090

E-mail: hk2005.yoon@gmail.com

This is an Open Access article distributed under the terms of the Creative Commons Attribution NonCommercial License (http://creativecommons.org/ licenses/by-nc/3.0/) which permits unrestricted noncommercial use, distribution, and reproduction in any medium, provided the original work is properly cited.

Copyright (C) 2014 Korean Society of Ultrasound in Medicine (KSUM)

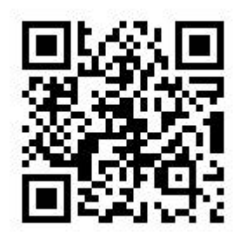

How to cite this article: Hwang JY, Yoon HK, Kim WK, Cho YA, Lee JS, Yoon CH, et al. Transperineal ultrasonography for evaluation of the perianal fistula and abscess in pediatric Crohn disease: preliminary study. Ultrasonography. 2014 Jul;33(3):184190. 
fistula, abscess, or similar lesions, and which is seen in 15\%-45\% of pediatric patients with CD [1-4]. The most common presentation of PCD is perianal fistulas with or without abscess, and which is followed by anal ulcer, skin tag, fecal incontinence, anal stenosis, and although very rarely, by anal cancer. In many patients, perianal disease constitutes the initial symptoms or signs of pediatric CD.

The primary goal of PCD treatment is improving and maintaining a patient's quality of life; therefore, correct diagnosis of perianal disease is essential for managing patients with CD [5]. The therapeutic plan for each patient should be devised considering the disease activity, location, and type of fistula, the presence or absence of an abscess, and the severity of the patient's symptoms. Various diagnostic modalities have been used to evaluate PCD. Although magnetic resonance imaging (MRI) and endoscopic ultrasonography (EUS) have been known to be the best modalities for depicting fistulas, several studies have shown transperineal ultrasonography (TPUS) to be a comparable alternative method for evaluating PCD, especially when considering its noninvasiveness, availability, and cost [6-8].

However, despite its advantages, TPUS is not widely used for evaluating PCD in pediatric patients, and there have been very few published reports regarding the clinical usefulness of TPUS for diagnosing pediatric CD. Therefore, the purpose of our study was to assess the feasibility and effectiveness of TPUS for evaluating PCD in pediatric patients.

\section{Materials and Methods}

This retrospective study was approved by our Institutional Review Board of the Asan Medical Center, and the requirement for informed consent was waived.

\section{Patients}

The clinical data regarding the pediatric patients with CD who underwent TPUS between September 2010 and August 2013 were retrospectively reviewed. All of the patients included in the study were diagnosed with CD by ileo-colonoscopy with biopsy and were less than 18 years of age. A total of 64 examinations were performed in 43 patients ( 34 males and nine females; mean age \pm standard deviation, $13.3 \pm 2.4$ years; age range, 6 to 17 years). All examinations were performed at a single medical institution by two board-certified pediatric radiologists (HKY and JYH with 19 and 3 years of experience, respectively). Seven patients underwent TPUS 3 times, 7 patients had 2 examinations, and the remaining 29 patients underwent a single examination.

All of the examinations were requested by pediatric gastroenterologists in order to identify a possible perianal fistula or abscess or to evaluate the status of the suspected PCD after medical or surgical treatment. Indications for the examination were categorized as screening or follow-up evaluation after conservative treatment or surgery. Surgical treatment for perianal diseases was performed within one year before or after TPUS.

\section{Technique}

The ultrasound examinations were performed using ultrasound units (IU-22, Phillips Medical Systems, Bothell, WA, USA) with 8-5 MHz curvilinear or 12-5 MHz linear transducers. The ultrasound probe was wrapped with a latex cover after applying contact gel on the surface of the probe. No patient preparation was required prior to the TPUS study.

Patients were asked to lie down in the left lateral decubitus position, and the ultrasound probe was then placed near the anal opening. Normal anatomy of the perianal region and scheme of TPUS are shown in Fig. 1. Patients were also asked if the procedure was tolerable or if they were experiencing pain or tenderness during the study. Their subjective pain description was classified on a scale of four: 0 , no pain; 1 , mild; 2 , moderate; and 4, severe pain. Ultrasound scanning was done in the sagittal and coronal planes in order to screen the perianal regions. The bladder was used as the anatomic landmark. The probe was angled to fully visualize the fistula once it was identified. The patient was asked to change position so as to assume the dorsal lithotomy position when necessary. In addition to 2-dimensional (D) grayscale imaging, a color Doppler study was performed in order to evaluate the vascularity of the fistulas and the anorectal canals.

\section{Imaging}

A fistulous tract was defined as an elongated or tubular hypoechoic structure with or without internal hyperechogenicity, and that started from the anal canal and extended to the perineal skin [9]. The perianal disease locations were categorized according to eight anatomic directions, that is, midline anterior, right anterior, right, right posterior, midline posterior, left posterior, left, and left anterior, centering on the anal canal. A fistula was considered to be active when it showed hyperemia along the fistulous tract on the color Doppler study and/or internal air bubbles on a 2D grayscale image, with or without fluid collection (Fig. 2) [9]. An abscess appeared as a hypoechoic, fluid-filled cavity in the perianal region (Fig. 3). Anal canal hyperemia was also evaluated on color Doppler examinations. Anal canal hyperemia was assessed subjectively during the examination and reviewed in consensus.

\section{Statistical Analysis}

Descriptive statistics were assessed to summarize the pain severity, 
location, and activity of a fistula, the presence of an abscess, and anal canal hyperemia. The Spearman rank correlation coefficient was calculated in order to assess the relationship of the pain severity and the activity of a fistula, the presence of an abscess, and anal canal hyperemia. Statistical analysis was conducted using IBM SPSS ver. 21. 0 (IBM Co., Armonk, NY, USA).

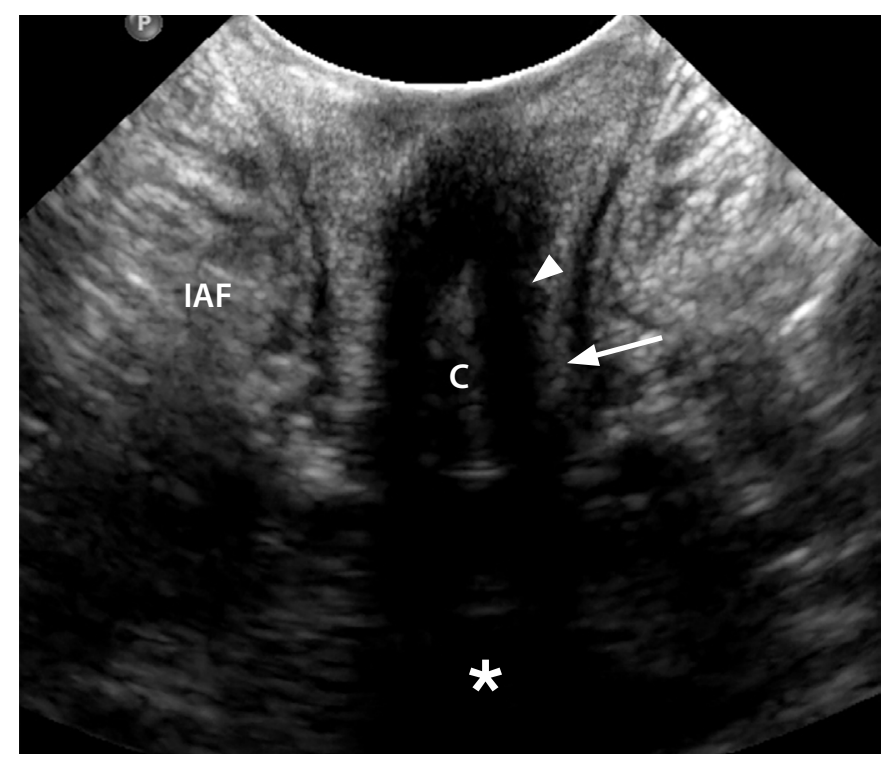
A

Fig. 1. Normal anal canal anatomy.

A coronal transperineal ultrasonogram (A) and schematic drawing (B) of the anal canal show a hypoechoic, internal anal sphincter (arrowhead) and a hyperechoic, external anal sphincter (arrow) on both sides of the anal canal (C). An ischioanal fossa (IAF) is located lateral to the external anal sphincter. The asterisk ${ }^{*}$ ) represents the urinary bladder.

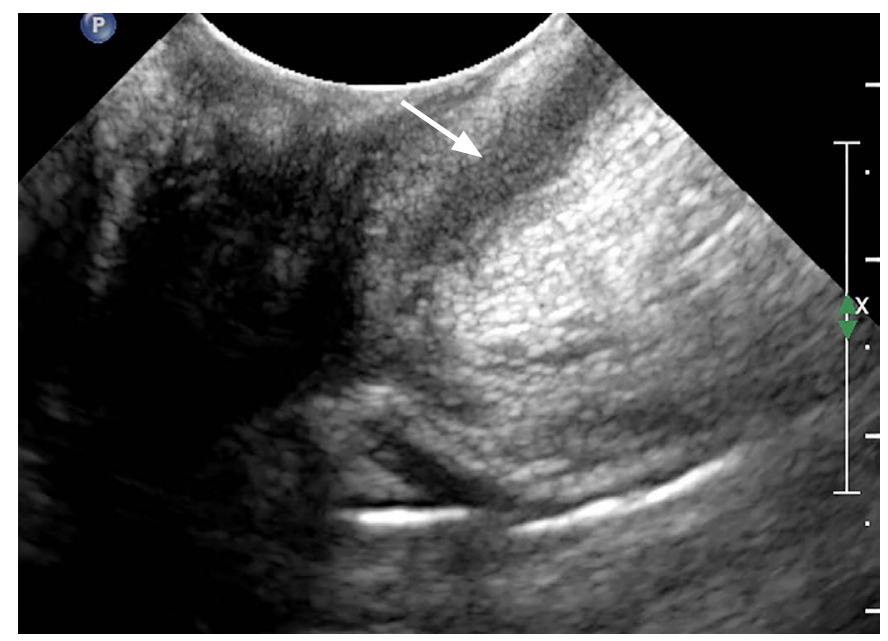

A

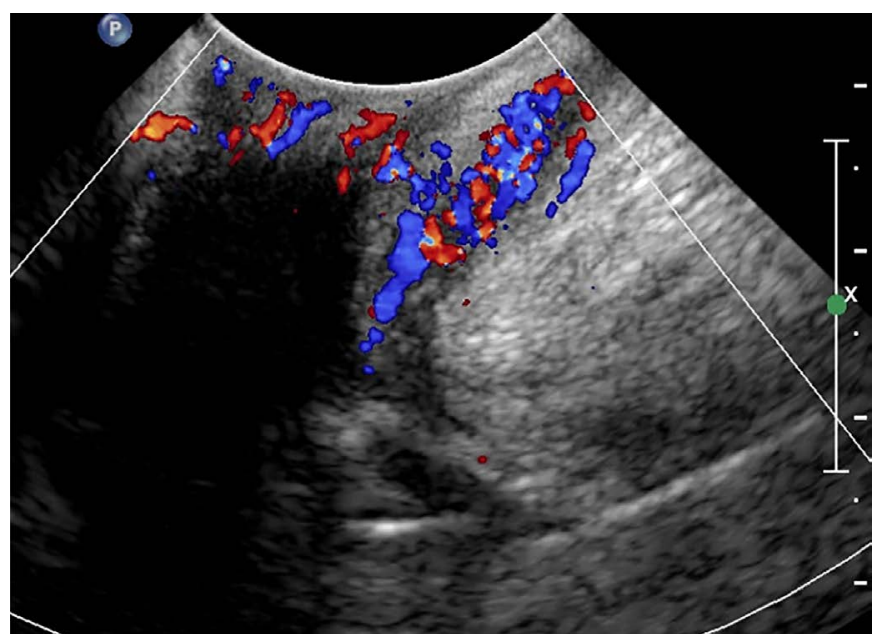

B

\section{Results}

All examinations were successfully performed without failure. No patient sedation was required. Among the 64 examinations, 23 were performed as part of the initial screening, 32 were for followup after a patient's medical treatment, and 9 were for follow-up following surgical treatment. A total of 62 fistulas were identified

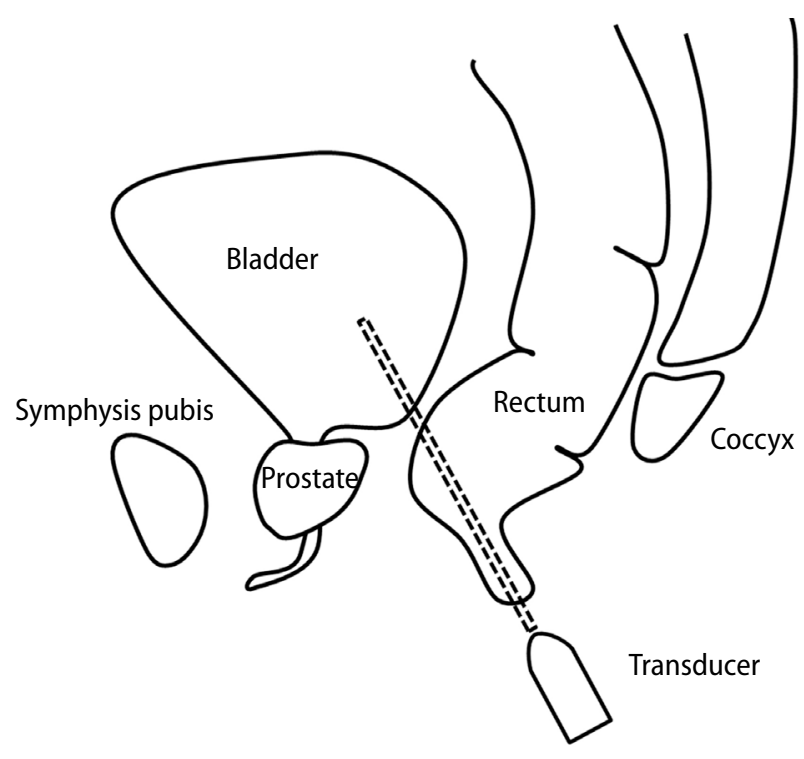

B

Fig. 2. A 15-year-old boy with an active fistula.

A. The grayscale image reveals a hypoechoic perianal fistula (arrow) extending to the perineal skin on the left side of the anal canal. B. This fistula shows marked hypervascularity on the color Doppler study. 


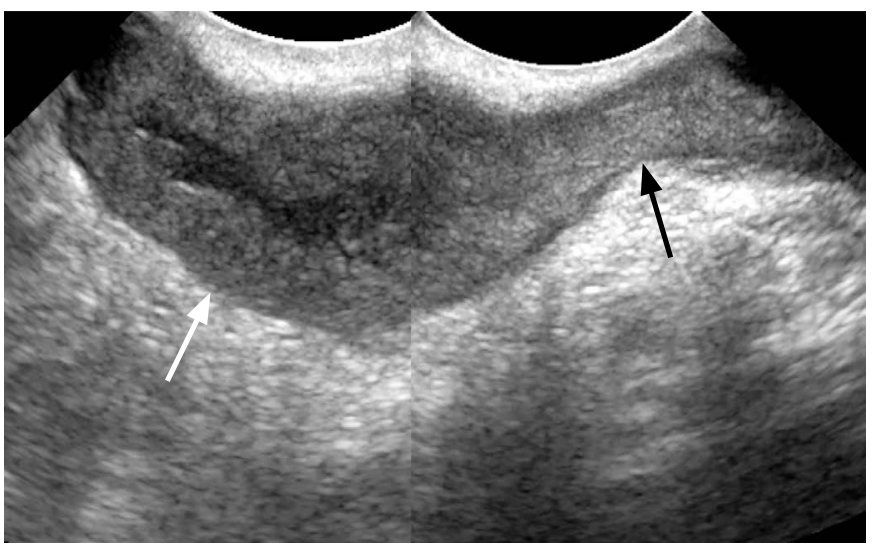

A

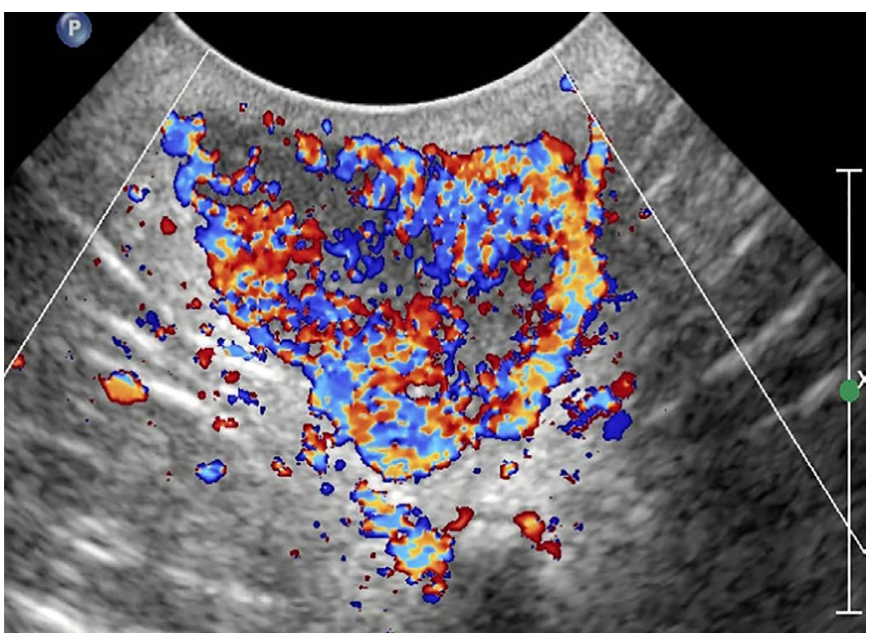

C

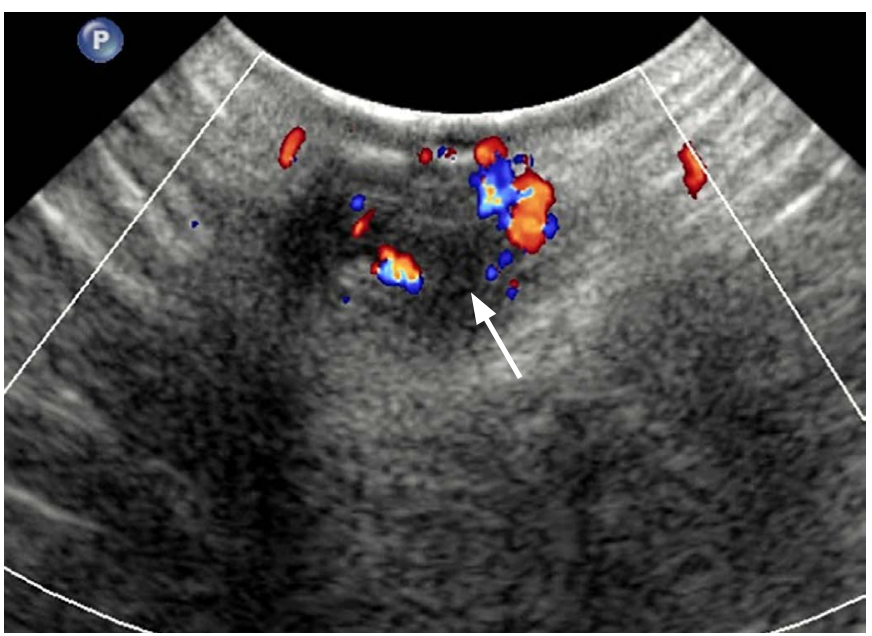

E

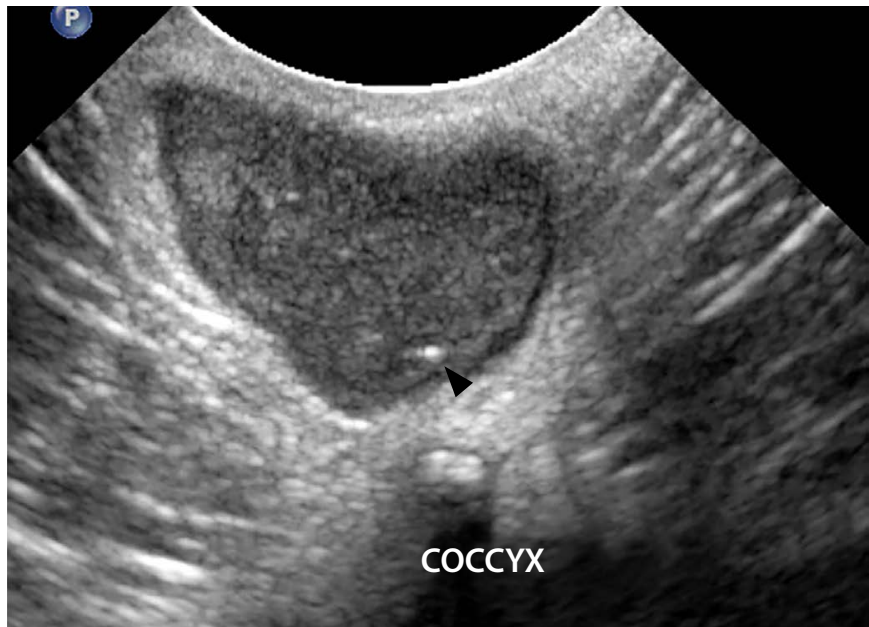

B

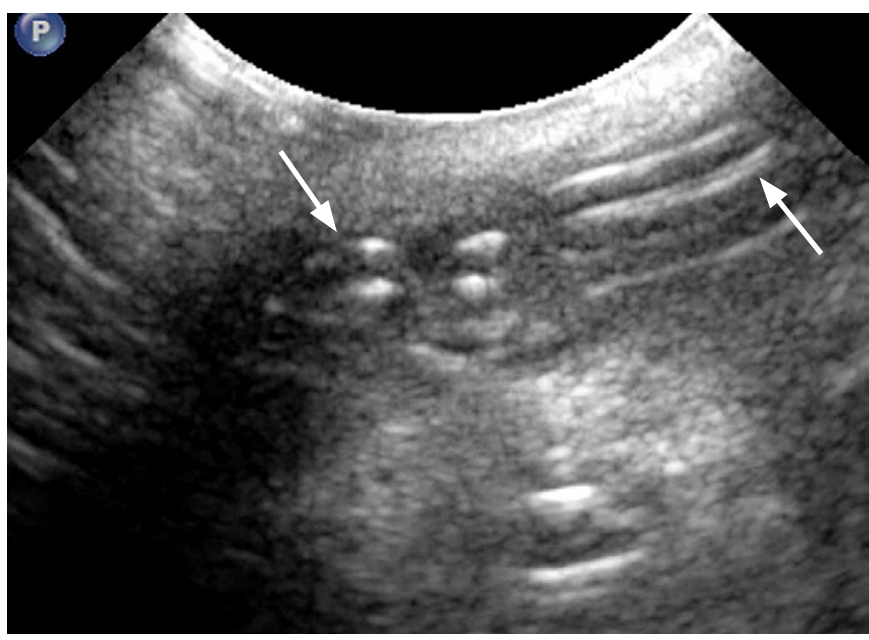

D

Fig. 3. A 15-year-old female with an active fistula and perineal abscess.

A, B. Grayscale images show an active fistula (black arrow) in the 7 $o^{\prime}$ clock direction of the anal canal and with an abscess cavity (white arrow). Note the air bubble within the abscess cavity (arrowhead). C. The color Doppler study reveals marked hypervascularity of the abscess and fistula. D, E. Follow-up transperineal ultrasonogram obtained in the same patient 19 months later. Surgical intervention was performed in the meantime. The grayscale image (D) shows the seton ribbon placed within the fistula (arrows). The color Doppler study (E) reveals the smaller abscess (arrow) with significantly decreased vascularity, compared to that seen in C. 
Table 1. Cross-correlation of the pain severity and fistula activity, the presence of an abscess, and anal-canal hyperemia

\begin{tabular}{llccc}
\hline & Variable & Active fistula & Abscess & Anal-canal hyperemia \\
\hline \multirow{2}{*}{ Pain } & Painless & 8 & 3 & 13 \\
& Mild & 16 & 6 & 14 \\
& Moderate & 5 & 2 & 3 \\
& Severe & 1 & $0.376(<0.01)$ & 1 \\
Coctive fistula & Correlation coefficient & $0.663(<0.01)$ & 9 & $0.363(0.01)$ \\
& Number & - & $0.271(0.03)$ & 21 \\
Abscess & Correlation coefficient & - & - & $0.405(<0.01)$ \\
& Number & - & - & $0.015(0.91)$ \\
\hline
\end{tabular}

The correlation coefficients represent the Spearman rank correlation coefficients. The data in parentheses represent the P-values.

in 46 examinations. Thirty-three examinations each demonstrated a single fistula, and 13 examinations showed more than 2 fistulas. Each of 18 examinations revealed no fistula.

TPUS examinations were well tolerated in the majority of the patients. Regarding the pain severity, 39 examinations (60.9\%) were performed without the patients experiencing any pain, 19 examinations (29.7\%) with mild pain, and 5 examinations (7.8\%) with moderate pain. Only one female (1.6\%) with an active fistula and abscess in the vulva complained of severe pain. The mean score of the degree of pain was $0.5 \pm 0.71$. The pain severity was moderately correlated with the fistula activity (correlation coefficient, $0.64 ; P<0.01$ ). Table 1 shows the results of the correlation of the pain severity and the fistula activity, the presence of an abscess, and anal-canal hyperemia. However, the degree of pain did not appear to be correlated with the presence of either an abscess or anal-canal hyperemia (correlation coefficients, 0.38 and 0.36 , respectively; $\mathrm{P}<0.05)$.

The perianal fistula locations were as follows: twenty-two fistulas $(22 / 62,35.5 \%)$ had a left anterior location, 16 fistulas (25.8\%) were in the right anterior, 11 fistulas (17.8\%) were in the right posterior, 10 fistulas $(16.1 \%)$ were in the left posterior, 2 fistulas (3.2\%) were in the right, and 1 fistula (1.6\%) was in the midline anterior. In our study, anterior fistulas were more common than posterior fistulas.

Thirty examinations (46.9\%) showed active fistulas. All active fistulas demonstrated hypervascularity on color Doppler ultrasound, and 7 examinations (23.3\%) revealed air bubbles within the fistula. Twelve examinations (18.8\%) revealed an abscess cavity, 9 of which $(75 \%)$ were associated with active fistulas. Anal canal hyperemia was seen in 31 examinations (48.4\%), in 21 of which (67.8\%) active fistulas were also seen.

Among the 14 patients who underwent TPUS more than twice, 2 patients had both active fistulas and abscesses, 2 patients had abscesses without fistulas, 7 patients had active fistulas without abscesses, 2 patients had silent fistulas, and 1 patient had no fistula or abscess. Of these 14 patients, 11 showed improvement of their active fistulas or abscesses. Among the two patients who had silent fistulas, one patient showed a new, active fistula and the other patient revealed no interval change of the presumed silent fistula seen on follow-up TPUS.

\section{Discussion}

The results of our preliminary study show the feasibility of TPUS for the evaluation of PCD in children and adolescents. Although the diagnostic performance could not be calculated due to the lack of a reference standard such as MRI or EUS, our study suggests that TPUS can be performed simply and without any preparation in order to evaluate perianal disease.

When performing TPUS, a broadband, $8-5 \mathrm{MHz}$, curved array transducer was used to outline the anatomy and the disease status of the perianal region. As this transducer has a small, convex head, it is suitable for evaluating the anal region. The penetration and resolution of this transducer were sufficient to complete the examinations in most of our patients. While a high-frequency, linear transducer may provide more detailed information, it has limited usefulness due to its relatively wide and rectangular head. Furthermore, it has limited penetration depth as it is short and with a maximum penetration depth of approximately $6 \mathrm{~cm}$ due to the high frequency of the transmitted pulse. In our experience, a highfrequency, linear-array transducer is advantageous for the evaluation of a superficial anal lesion not located at the anal opening.

Currently, the diagnosis of PCD is usually made by examination with the patient under anesthesia (EUA), by EUS, and by MRI [10]. EUA is known as the gold standard as it allows direct visualization, palpation, and probing of a fistula [9]. It is a useful tool not only for 
identifying and classifying perianal fistulas, but also for monitoring a patient's treatment response [11]. Its invasiveness and need for sedation might be optimal for pediatric patients. MRI has become the primary imaging modality used to detect and classify $P C D$, and its performance has been reported to be comparable to that of EUS and EUA [12-14]. MRI can provide detailed anatomical information regarding the sphincter complex and perianal inflammatory disease with high soft-tissue contrast using either an endorectal coil or an external surface coil. However, it is expensive, requires more time to obtain images, and has the potential risks associated with the use of contrast material.

TPUS is a simple, cost-effective, and less invasive diagnostic modality which is comparable to MRI and EUS for evaluating PCD. Recent studies have reported that TPUS is accurate for detecting perianal fistulas and perianal abscesses $[6,7,8,15]$. In the published medical literature, the sensitivity, specificity, and positive predictive value of TPUS for detecting fistulas has been reported to be $84.9 \%-100 \%, 80 \%-100 \%$, and $86.5 \%$, respectively $[6-8,16]$. The detectability of TPUS increased slightly in combination with a color Doppler study [7].

In addition, TPUS could also prove to be a useful imaging modality for monitoring the treatment response by assessing the disease activity [17]. Determination of the activity is paramount in planning the treatment of PCD. Management of perianal fistulas and abscesses includes both medical and surgical treatment. Once the possibility of perianal sepsis is excluded, active fistulas and abscesses should be treated medically, as surgical management can result in poor wound healing. Antibiotics are the first-line therapy for medical treatment in conjunction with immunosuppressant medications such as azathioprine, cyclosporine, and infliximab [18].

Although perianal abscess justifies emergency surgical management, fistulotomy should be initiated carefully according to the complexity of the fistula and the disease activity. Seton is a nonabsorbable, string-like material such as nylon or rubber. Placement of the seton through the fistulous tract may help to control the perianal disease by keeping the fistula open for continuous drainage of any infection. It can also be a successful long-term solution even in the case of a complex fistula accompanied by active proctitis $[18,19]$. In our study, the fistula activity was assessed by identifying the hypervascularity at the fistula wall as well as any air bubbles within the fistula or abscess. Similarly, we assumed that there was active proctitis when the anal canal showed hyperemia on a color Doppler study.

In our study, the pain severity was correlated with the presence of active disease, that is, all patients who complained of moderate or severe pain had active disease and anal-canal hyperemia. On the other hand, the majority of patients who had no active disease or abscess seen on TPUS complained of no pain or mild tenderness during the ultrasound scanning. Therefore, TPUS can be used as a simple and safe imaging modality for evaluating pediatric CD.

TPUS has limitations regarding the identification and classification of fistulas located in deeper spaces of the perianal region. Although several studies have suggested that TPUS can be a useful modality for identifying and classifying fistulas $[15,16]$, in our clinical experience, it was difficult to determine the location of the internal opening and classify the fistula type according to Park's classification. This is why MRI is recommended in order to plan the surgical treatment and to evaluate the pelvic extension of inflammatory processes.

Our study did not have a reference standard such as MRI or EUS. Nor was the disease activity validated either by another imaging study or by clinical factors. This retrospective and preliminary study was designed to assess the applicability and feasibility of TPUS in pediatric patients. Although most of our patients did not undergo pelvic MRI or EUS, TPUS findings helped clinicians to determine each patient's management plan. Further studies are required to address the diagnostic accuracy compared with the reference standard for assessing $P C D$.

In conclusion, TPUS is a feasible and safe imaging modality for evaluating PCD in pediatric patients. Color Doppler studies with TPUS are very useful for demonstrating the increased vascularity along the fistula or abscess, which may represent inflammatory activity.

ORCID: Jae-Yeon Hwang: http://orcid.org/0000-0003-2777-3444; Hye-Kyung Yoon: http://orcid.org/0000-0001-7547-0320; Wee Kyoung Kim: http://orcid.org/00000002-0441-8493; Young Ah Cho: http://orcid.org/0000-0002-3474-8653; Jin Seong Lee: http://orcid.org/0000-0002-8470-4595; Chong Hyun Yoon: http://orcid.org/00000001-8642-6130; Yeoun Joo Lee: http://orcid.org/0000-0001-8012-5433; Kyung Mo Kim: http://orcid.org/0000-0001-7896-6751

\section{Conflict of Interest}

No potential conflict of interest relevant to this article was reported.

\section{Acknowledgments}

This study was supported in part by the Research Fund of the Korean Society of Ultrasound in Medicine.

\section{References}

1. Keljo DJ, Markowitz J, Langton C, Lerer T, Bousvaros A, Carvalho R, et al. Course and treatment of perianal disease in children newly diagnosed with Crohn's disease. Inflamm Bowel Dis 2009;15:383387.

2. Kugathasan S, Judd RH, Hoffmann RG, Heikenen J, Telega G, Khan 
$\mathrm{F}$, et al. Epidemiologic and clinical characteristics of children with newly diagnosed inflammatory bowel disease in Wisconsin: a statewide population-based study. J Pediatr 2003;143:525-531.

3. Markowitz J, Daum F, Aiges H, Kahn E, Silverberg M, Fisher SE. Perianal disease in children and adolescents with Crohn's disease. Gastroenterology 1984;86(5 Pt 1):829-833.

4. Burbige EJ, Huang SH, Bayless TM. Clinical manifestations of Crohn's disease in children and adolescents. Pediatrics 1975;55: 866-871.

5. Becker SA. Perianal Crohn's disease. Isr Med Assoc J 2007;9:495.

6. Wedemeyer J, Kirchhoff T, Sellge G, Bachmann O, Lotz J, Galanski M, et al. Transcutaneous perianal sonography: a sensitive method for the detection of perianal inflammatory lesions in Crohn's disease. World I Gastroenterol 2004;10:2859-2863.

7. Mallouhi A, Bonatti $H$, Peer S, Lugger P, Conrad F, Bodner G. Detection and characterization of perianal inflammatory disease: accuracy of transperineal combined gray scale and color Doppler sonography. J Ultrasound Med 2004;23:19-27.

8. Stewart LK, McGee J, Wilson SR. Transperineal and transvaginal sonography of perianal inflammatory disease. AJR Am J Roentgenol 2001;177:627-632.

9. Ardizzone S, Maconi G, Cassinotti A, Massari A, Porro GB. Imaging of perianal Crohn's disease. Dig Liver Dis 2007;39:970-978.

10. Schwartz DA, Wiersema MJ, Dudiak KM, Fletcher JG, Clain JE, Tremaine WJ, et al. A comparison of endoscopic ultrasound, magnetic resonance imaging, and exam under anesthesia for evaluation of Crohn's perianal fistulas. Gastroenterology 2001;121: 1064-1072.

11. Lahat A, Assulin Y, Beer-Gabel M, Chowers Y. Endoscopic ultrasound for perianal Crohn's disease: disease and fistula characteristics, and impact on therapy. J Crohns Colitis 2012;6:311-316.

12. Buchanan GN, Halligan S, Bartram Cl, Williams AB, Tarroni D, Cohen CR. Clinical examination, endosonography, and MR imaging in preoperative assessment of fistula in ano: comparison with outcome-based reference standard. Radiology 2004;233:674-681.

13. Orsoni P, Barthet M, Portier F, Panuel M, Desjeux A, Grimaud JC. Prospective comparison of endosonography, magnetic resonance imaging and surgical findings in anorectal fistula and abscess complicating Crohn's disease. Br J Surg 1999;86:360-364.

14. Beets-Tan RG, Beets $G L$, van der Hoop AG, Kessels AG, Vliegen RF, Baeten $C G$, et al. Preoperative MR imaging of anal fistulas: does it really help the surgeon? Radiology 2001;218:75-84.

15. Zbar AP, Oyetunji RO, Gill R. Transperineal versus hydrogen peroxide-enhanced endoanal ultrasonography in never operated and recurrent cryptogenic fistula-in-ano: a pilot study. Tech Coloproctol 2006;10:297-302.

16. Maconi G, Ardizzone S, Greco S, Radice E, Bezzio C, Bianchi Porro $G$. Transperineal ultrasound in the detection of perianal and rectovaginal fistulae in Crohn's disease. Am J Gastroenterol 2007; 102:2214-2219.

17. Rasul I, Wilson SR, MacRae H, Irwin S, Greenberg GR. Clinical and radiological responses after infliximab treatment for perianal fistulizing Crohn's disease. Am J Gastroenterol 2004;99:82-88.

18. Safar B, Sands D. Perianal Crohn's disease. Clin Colon Rectal Surg 2007;20:282-293.

19. White RA, Eisenstat TE, Rubin RJ, Salvati EP. Seton management of complex anorectal fistulas in patients with Crohn's disease. Dis Colon Rectum 1990;33:587-589. 